\title{
L-DOPA improves extinction memory retrieval after successful fear extinction
}

\author{
A. M. V. Gerlicher ${ }^{1,2,3}$ (D) 0 . Tüscher ${ }^{2,4} \cdot$ R. Kalisch ${ }^{1,2}$
}

Received: 6 February 2019 / Accepted: 5 June 2019 / Published online: 26 June 2019

(C) The Author(s) 2019

\begin{abstract}
Rationale A promising strategy to prevent a return of fear after exposure-based therapy in anxiety disorders is to pharmacologically enhance the extinction memory consolidation presumed to occur after exposure. Accumulating evidence suggests that the effect of a number of pharmacological consolidation enhancers depends on a successful fear reduction during exposure. Here, we employed the dopamine precursor L-DOPA to clarify whether its documented potential to enhance extinction memory consolidation is dependent on successful fear extinction.

Methods In two double-blind, randomized and placebo-controlled experiments (experiment 1: $N=79$, experiment 2: $N=32$ ) comprising fear conditioning (day 1 ), extinction followed by administration of $150 \mathrm{mg}$ L-DOPA or placebo (day 2 ) and a memory test (day 3 ) in healthy male adults, conditioned responses were assessed as differential skin conductance responses. We tested whether the effect of L-DOPA on conditioned responses at test depended on conditioned responses at the end of extinction in an experiment with a short (10 trials, experiment 1$)$ and long (25 trials, experiment 2 ) extinction session.

Results In both experiments, the effect of L-DOPA was dependent on conditioned responses at the end of extinction. That is, postextinction L-DOPA compared to placebo administration reduced conditioned responses at test only in participants showing a complete reduction of conditioned fear at the end of extinction.

Conclusion The results support the potential use of L-DOPA as a pharmacological adjunct to exposure treatment, but point towards a common boundary condition for pharmacological consolidation enhancers: a successful reduction of fear in the exposure session.
\end{abstract}

Keywords Dopamine - Fear conditioning · Extinction · Memory consolidation · Anxiety · Post-traumatic stress disorder · Exposure treatment $\cdot$ Cognitive-behavioural therapy

Electronic supplementary material The online version of this article (https://doi.org/10.1007/s00213-019-05301-4) contains supplementary material, which is available to authorized users.

A. M. V. Gerlicher

a.m.v.gerlicher@uva.nl

1 Neuroimaging Center (NIC), Focus Program Translational Neuroscience (FTN), Johannes Gutenberg University Medical Center, Langenbeckstr. 1, 55131 Mainz, Germany

2 Deutsches Resilienz Zentrum (DRZ), Johannes Gutenberg University Medical Center, Untere Zahlbacher Str. 8, 55131 Mainz, Germany

3 Present address: Department of Clinical Psychology, University of Amsterdam, Nieuwe Achtergracht 129B, 1018 WS Amsterdam, The Netherlands

4 Department of Psychiatry and Psychotherapy, Johannes Gutenberg University Medical Center, Untere Zahlbacher Str. 8, 55131 Mainz, Germany

\section{Introduction}

Exposure-based treatments are rooted in the principles of fear extinction and are an initially very effective intervention to reduce pathological fear and anxiety in the context of cognitive-behavioural therapy (Beck et al. 2005). In exposure-based treatments, patients are asked to confront themselves with the threat-associated stimulus or situation. The experience that no actual harm occurs during the exposure to the feared stimulus or situation results in a reduction of pathological fear and anxiety (Craske et al. 2008; Craske et al. 2014). Similarly, during fear extinction in the laboratory a conditioned stimulus (CS) that was previously paired with an aversive unconditioned stimulus (US) is repeatedly presented in the absence of the US, resulting in a reduction of conditioned fear responses (CRs). In both laboratory settings and in clinical practice CRs can, however, be re-evoked 
despite successful extinction learning (Bouton 2004). The occurrence of a return of fear after extinction indicates that extinction learning does not erase the initial 'CS-US' association (fear memory), but rather creates a competing 'CS-noUS' association, or extinction memory, that is suggested to inhibit the expression of fear (Bouton 2004). Thus, in order to ensure a reliable long-term reduction of fear after extinction learning or exposure treatment, the extinction memory trace has to be reliably retrieved upon a new encounter with the CS despite competition with the fear memory trace.

One strategy to facilitate extinction over fear memory retrieval is to enhance the strength of extinction memories. To this end, recent studies (Fitzgerald et al. 2014; Singewald et al. 2015) have investigated pharmacological interventions targeting either extinction learning itself or the extinction memory consolidation presumed to occur in the hours after new learning (McGaugh, 2000). The NMDA receptor agonist D-cycloserine (DCS) is among the best-studied pharmacological adjuncts to exposure therapy (Otto et al. 2016). Interestingly, animal studies in which DCS was administered either before or after extinction learning point towards a potential complication in pharmacological extinction memory enhancement. Namely, DCS was only beneficial in animals exhibiting low fear at the end of extinction (Weber et al. 2007; Bouton et al. 2008; Bolkan and Lattal 2014). Experimental manipulations of extinction duration revealed that only a combination of DCS with long extinction resulted in improved extinction memory retrieval, whereas DCS administration after short extinction did either have no effect (Bouton et al. 2008) or even led to greater fear responses at test (Lee et al. 2006). It was suggested that a short extinction session during which CRs remain high may merely evoke a reactivation of the fear memory trace (Merlo et al. 2014). Upon reactivation, fear memories become malleable and labile again. For memories to persist after and beyond reactivation, they must be stabilized by fear memory reconsolidation processes (Nader et al. 2000). Administering DCS after short extinction may, thus, have enhanced the fear memory reconsolidation and strengthened the fear memory. In contrast, during a long extinction session during which CRs are completely reduced, an extinction memory trace may have been acquired successfully and a subsequent DCS administration may have enhanced extinction memory consolidation as desired. Thus, the behavioural expression of fear at the end of an extinction session may be an indicator of the currently active memory trace that is susceptible to pharmacological enhancement (Eisenberg et al. 2003; King et al. 2018). Analogous observations have been made in patient studies combining exposure treatment with DCS (Smits et al., 2013a, b; but see de Kleine et al. 2015), the $\alpha 2$-adrenergic agonist yohimbine (Smits et al. 2014) or the neuro-metabolic enhancer methylene blue (Telch et al. 2014) across different anxiety disorders. Also in patients, the effects of DCS, yohimbine or methylene blue were a function of subjective fear at the end of exposure.
Together with others, we recently introduced L-DOPA, a dopamine precursor, as a new enhancer of extinction memory consolidation. Post-extinction L-DOPA administration in rodents improved extinction memory retrieval, reducing the return of fear after the mere passage of time ('spontaneous recovery'), after an unannounced re-confrontation with the US ('reinstatement') and even after confrontation with the CS in a context different from the extinction context (Haaker et al. 2013; Whittle et al. 2016), a phenomenon called 'contextual renewal of fear'. In two MRI studies, we translated these findings to humans (Haaker et al. 2013; Gerlicher et al. 2018) and identified a mechanism via which dopamine enhances human extinction memory consolidation. Namely, post-extinction L-DOPA administration increased spontaneous reactivations of an extinction learningrelated prefrontal activity pattern in the hours after extinction learning (Gerlicher et al. 2018). Interestingly, in these data, we also observed a weak relationship between conditioned responses at the end of extinction and extinction memory retrieval after L-DOPA intake (Gerlicher et al. 2018). Even though only trend-wise significant, this observation indicates that - similar to DCS, yohimbine and methylene blue - memory consolidation enhancement by L-DOPA may also depend on a successful reduction of fear at the end of extinction.

Here, we aimed to clarify whether the effect of a postextinction L-DOPA administration is indeed dependent on extinction success. We conducted two experiments with conditioning (in context $\mathrm{A}$ ) on day 1 , extinction (in context $\mathrm{B}$ ) and subsequent administration of either L-DOPA or placebo on day 2 and a test of extinction memory retrieval (in the extinction context B) on day 3. In experiment 1, a short extinction session (10 trials) was employed with the intention to assure sufficient interindividual variability in CRs at the end of extinction due to incomplete extinction learning in some subjects but not in others (Fig. 1a). Given high inter-individual variability in extinction

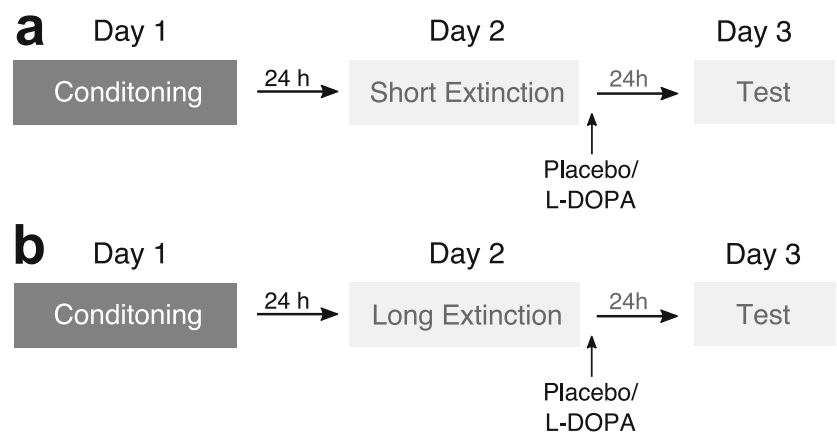

Fig. 1 Experimental design of experiment 1 and 2. a In order to test the potential influence of inter-individual differences in extinction success on the effect of a post-extinction administration of L-DOPA on extinction memory retrieval, we aimed to increase inter-individual variability in extinction success by reducing the number of trials during extinction compared to a previous study (Gerlicher et al. 2018) from 15 to $10 \mathrm{CS}+$ and CS- trials in experiment 1 . b In experiment 2, we aimed to induce successful extinction learning experimentally by increasing the number of trials during the extinction session from 15 in a previous study (Gerlicher et al. 2018) to 25 CS+ and CS- trials (long extinction) in experiment 2 
success, we expected to find that the effect of L-DOPA on conditioned fear at test would be dependent on conditioned fear responses at the end of extinction. In experiment 2, we aimed to extend the correlative evidence from experiment 1 by increasing the number of extinction trials (long extinction, 25 trials) in order to produce low differential CRs at the end of extinction on day 2 consistently across participants (Fig. 1b). We hypothesised that after successful extinction a post-extinction L-DOPA compared to placebo administration would significantly reduce conditioned fear at test. In line with our previous studies (Haaker et al. 2013; Haaker et al. 2015; Gerlicher et al. 2018), we assessed conditioned responses (CRs) as skin conductance response (SCR) to the reinforced CS+ compared to an unreinforced CS-. With regard to the commonly reported differential effects of pharmacological memory manipulations on different measures of conditioned fear in humans (Kindt et al. 2009; Soeter and Kindt 2010, 2011, 2012; Sevenster et al. 2012, 2014), we also assessed fear-potentiated startle responses (FPS) and online fear ratings to CS+ and CS- as secondary outcome measures.

\section{Materials and methods}

\section{Participants}

In total, 80 healthy male participants took part in experiment 1. One participant had to be excluded due to recording software problems, resulting in a sample size of 79 (mean \pm SD age, $27 \pm 2$ years) in experiment 1 . In experiment 2 , we tested 32 (mean $\pm \mathrm{SD}$ age, $28 \pm 3$ years) healthy male participants. In experiment 2 , the required sample size was estimated based on the effect of L-DOPA on differential SCR at test in successful extinguishers in experiment 1 using $\mathrm{G}^{*}$ Power (Faul et al. 2007; stim*group partial $\eta^{2}=.29$, power $1-\beta=.80$, correlation among repeated measures $r=.50$ ) and further increased in order to compensate for the low signal-to-noise ratio of psychophysiological measures. We restricted recruitment to male participants as the estrous cycle interacts with extinction memory consolidation (Lebron-Milad and Milad 2012; Cover et al. 2014) and dopamine can have opposing effects on extinction depending on estrous cycle phase (Rey et al. 2014). A boardcertified physician screened participants for contraindications of L-DOPA intake, current physiological, neurological or psychiatric disorders, excessive consumption of nicotine ( $>10$ cigarettes/day), alcohol (>15 glasses of beer/wine per week) or cannabis ( $>1$ joint/month), participation in other pharmacological studies and tinnitus (as contraindication for startle probe exposure). Drug abuse was assessed via urine test (M-10/3DT; Diagnostik Nord, Schwerin, Germany). During the screening session, we also tested skin conductance responding in each participant. To this aim, we attached two electrodes of the eSense skin response device (Mindfield ${ }^{\circledR}$
Biosystems Ltd., Berlin, Germany) to the medial phalanges of the index and middle finger. Participants were asked to take several deep breaths. In addition, the physician clapped his hands without announcement. Both deep breathing and acoustic startle usually result in a deflection of the skin conductance, not seen in skin conductance non-responding individuals. None of the participants screened for the present experiments had to be excluded according to this criterion. Both experiments were approved by the local ethics committee (Ethikkommission der Landesärztekammer, RhinelandPalatinate, Germany) and conducted in accordance with the Declaration of Helsinki.

\section{Stimuli}

Two black geometric symbols (triangle, circle) served as CSs. The CSs were presented in the centre of a computer screen and super-imposed on background pictures of either a kitchen or a living room, which served as context A or B. Assignment of symbols to $\mathrm{CS}+/ \mathrm{CS}$ - and rooms to contexts $\mathrm{A} / \mathrm{B}$ was counterbalanced between participants and groups. A painful electrical stimulation consisting of three square-wave pulses $(50 \mathrm{~ms}$ inter-stimulus interval) of $2 \mathrm{~ms}$ was employed as US. Pain stimuli were generated by a DS7A electrical stimulator (Digitimer Ltd., Welwyn Garden City, UK) and delivered on the right dorsal hand through a surface electrode with platinum pin (Specialty Developments, Bexley, UK).

\section{Experimental procedure}

\section{Day 1 -conditioning}

Upon arrival, participants completed questionnaires on trait and state anxiety (STAI-T/STAI-S; Spielberger et al. 1970), anxiety sensitivity (ASI-3; Taylor et al. 2007) and demographic data. Subsequently, electrodes were attached and US intensity was calibrated to a level rated as 'maximally painful, but still tolerable'. Familiarization consisted of two CS presentations in both contexts and a practice rating of fear and US expectancy. Before the start of the actual experiment, participants were instructed that the experiment would be distributed across 3 days, that one symbol would never be followed by an electric shock and that their task was to find out what rule applied to the other symbol. The experiment started every day with US expectancy ratings for each CS, followed by a habituation phase. During the habituation phase participants were presented with $30 \mathrm{~s}$ background noise and 10 startle probes (noise alone trials, NA), during which the context was already presented on the computer screen. The context remained on the screen continuously throughout the experiment. Participants were asked to rate their subjective fear/distress/tension within the first $5 \mathrm{~s}$ of the $\mathrm{CS}$ presentation. The CS was presented for $8 \mathrm{~s}$ in total. Startle probes were 
delivered $7.4 \mathrm{~s}$ after $\mathrm{CS}$ onset. In case of reinforced $\mathrm{CS}+$ presentations, USs were delivered $500 \mathrm{~ms}$ later. Inter-trial intervals (ITI) lasted 15, 20 or $25 \mathrm{~s}$ (mean of $20 \mathrm{~s}$ ). Trial order was randomized and not more than two trials of the same type (i.e. CS+, CS-, NA) succeeded each other. During conditioning, participants were presented with $5 \mathrm{CS}+, 5 \mathrm{CS}$ - and 5 NA trials in context $\mathrm{A}$ in both experiments 1 and 2 . In both experiments, four out of $5 \mathrm{CS}+$ presentations (i.e. 80\%) were reinforced. After conditioning, participants again rated their US expectancy. The total duration of the session amounted to approximately $1 \mathrm{~h}$ on day 1 .

\section{Day 2-extinction}

The extinction session took place approximately $24 \mathrm{~h}( \pm 1 \mathrm{~h})$ after conditioning. Upon arrival participants were asked to fill out the STAI-S. We did not re-calibrate the US, but informed participants that their individual US strength from day 1 would be applied and that the experiment would continue. In experiment 1 , the extinction session consisted of $10 \mathrm{CS}+, 10$ CS- and 10 NA trials presented in context B. In experiment 2 , the extinction session consisted of $25 \mathrm{CS}+, 25 \mathrm{CS}-$ and $25 \mathrm{NA}$ trials presented in context $\mathrm{B}$. After the extinction session electrodes were detached and participants were administered either a placebo or L-DOPA pill. After pill intake, participants remained under observation for $1 \mathrm{~h}$ while blood pressure and heart rate were monitored regularly. Participants then filled out an L-DOPA side effects questionnaire and the STAI-S. The total duration of the session amounted to approximately $2.5 \mathrm{~h}$ on day 2.

\section{Day 3-test}

The test session took place approximately $24 \mathrm{~h}( \pm 1 \mathrm{~h})$ after the extinction session. Upon arrival, participants were asked to fill out the side effects questionnaire and the STAI-S. After electrode attachment, participants were again only instructed that the experiment would continue and presented with $8 \mathrm{CS}+, 8$ CS- and 8 NA trials in context B in both experiments 1 and 2 . The total duration of the session amounted to approximately $45 \mathrm{~min}$ on day 3 .

\section{Drug treatment}

Participants were asked to refrain from eating, drinking and smoking for $2 \mathrm{~h}$ prior to drug intake. Participants were randomly assigned to the L-DOPA or placebo group with the restriction that groups were matched on STAI-T and ASI scores. Assignment of participants to the placebo or LDOPA group was conducted by a person not involved in data collection. Participants were either administered 150/37.5 mg levodopa-benserazide (Levodopa-Benserazid-ratiopharm $®$, Germany; for dosage, see (Haaker et al. 2013, 2015) or an identically looking capsule filled with mannitol and aerosol (placebo). Drugs were prepared and provided by the pharmacy of the University Medical Center Mainz and administered double-blind.

\section{Skin conductance response}

Electrodermal activity was recorded from the thenar and hypothenar of the left hand using self-adhesive $\mathrm{Ag} / \mathrm{AgCl}$ electrodes (EL-509, BIOPAC® Systems Inc., Goleta, CA, USA). The raw signal was amplified and low-pass filtered with a cutoff frequency of $1 \mathrm{~Hz}$. Using a custom-made analysis script, we manually scored the first local minimum in the skin conductance time course in a time window from 900 to $4000 \mathrm{~ms}$ after CS onset as response onset (Boucsein et al. 2012) and the following local maximum as response peak (onset to peak latency 500-4000 ms). We assessed the amplitude of an SCR then as onset-to-peak difference. Critically, the experimenter scoring the data was blinded to both stimulus type (CS+/CS-) of each SCR and group belongingness (placebo/ L-DOPA) of each participant. Responses smaller than $0.02 \mu \mathrm{s}$ were scored as zero and remained in the analysis. If more than $75 \%$ of trials had to be scored as zero, data of that subject/day was considered invalid. In experiment 1 , this applied to $n=3$ / 2/6 (day 1/2/3) participants, in experiment 2 to $n=1 / 0 / 2$ participants, leaving $N=35 / 35 \mathrm{~L}-\mathrm{DOPA} /$ placebo complete data sets in experiment 1 and $N=16 / 14$ L-DOPA/placebo data sets in experiment 2 for statistical analysis. To normalize distributions, data were log-transformed (after a constant of 1 was added) and range-corrected within subject and day (Lykken and Venables 1971).

\section{Fear-potentiated startle response}

The eye blink reflex was elicited by a loud noise $(40 \mathrm{~ms} /$ $104 \mathrm{~dB})$ presented against constant broadband noise $(70 \mathrm{~dB})$ via headphones (Sennheiser, HD 380 pro). Two 7-mm Ag/ $\mathrm{AgCl}$ electrodes filled with electrolyte gel (Signa Gel, Parker) were positioned approximately $1 \mathrm{~cm}$ below the pupil and $1 \mathrm{~cm}$ below the lateral canthus, the outer corner of the right eye (Fridlund and Cacioppo 1986). We recorded the electromyographic signal using the BIOPAC MP150 and the EMG100C device. The signal was amplified and band-pass filtered between 10 and $500 \mathrm{~Hz}$ and offline rectified and integrated with a time constant of $10 \mathrm{~ms}$ using a custom-made analysis script. FPS amplitudes were scored as the difference between response onset and maximum of responses elicited within 20-120 ms after startle probe onset. If a session comprised more than $75 \%$ of trials affected by artefacts or blinks, data from that subject/day was considered invalid. This applied to $N=8 / 2 / 7$ (day $1 / 2 / 3$ ) participants in experiment 1 and $N=1$ participant on day 3 in experiment 2 , leaving $N=31 / 32$ L-DOPA/placebo (experiment 1) and $N=15 / 16 \mathrm{~L}-\mathrm{DOPA} /$ 
placebo (experiment 2) complete data sets for statistical analysis. Data of each participant were standardized per day (zscore) and linearly transformed to $T$-scores.

\section{Fear and US expectancy ratings}

Within the first $5 \mathrm{~s}$ of each CS presentation participants were asked to indicate their fear/distress/tension on a visual analogue scale ranging from $0=$ 'no fear/distress/tension' to $100=$ 'high fear/distress/tension' with a mouse-button click. If participants did not respond within this time window, missing values were replaced by subject- and day-specific means. Before and after each experimental phase, participants were asked to indicate the expectancy of receiving an electric shock for each CS on a scale from $0=$ 'no expectancy' to $100=$ 'high expectancy'.

\section{Statistical analysis}

For statistical analysis of experimental and group effects on SCRs, we operationalized CRs at the end of conditioning (i.e. two trials in experiment 1 and 2) and CRs at the beginning or end (i.e. two trials in experiment 1, five trials in experiment 2) of extinction, by averaging SCRs to CS+ and CS- over $20 \%$ of trials at the beginning or end of the respective experimental phase. This procedure was a-priori standardized with the aim to harmonize measures across experiments with varying trial numbers (Gerlicher et al. 2018). As in previous studies (Haaker et al. 2013, 2015; Gerlicher et al. 2018), the effect of L-DOPA on extinction memory retrieval was tested on SCRs elicited by CS+ and CS- averaged across the whole test phase on day 3 (i.e. eight trials in both experiments 1 and 2). These apriori determined operating procedures are an attempt to prevent post hoc biases in operationalization decisions (Ney et al. 2018). Please see Fig. 2 for single-trial SCRs in both experiments.

Repeated measures ANOVA with stimulus (CS+/CS-) as within- and group (placebo/L-DOPA) as between-subject factor was employed to assess whether the placebo and L-DOPA group differed in CRs at the end of conditioning, the beginning or the end of extinction and to test the potential effect of postextinction L-DOPA administration on CRs at test on day 3 on SCR data. To specifically test the hypothesis that the effect of LDOPA on conditioned fear at test on day 3 was dependent conditioned fear at the end of extinction on day 2, multiple linear regression analysis was performed with differential CRs (SCR $\mathrm{CS}+>\mathrm{CS}-$ ) at the end of extinction, group (placebo/L-DOPA) and their interaction as independent and differential CRs (SCR $\mathrm{CS}+>\mathrm{CS}-$ ) at test as dependent variable. We also included differential CRs at the end of conditioning and the beginning of extinction as covariates into the regression analysis as extinction success could have been confounded by initial fear acquisition or fear at the beginning of extinction.
Statistical analysis of FPS, online fear and US expectancy rating data followed the same procedure (for results see Supplementary Fig. 1-6). All statistical tests were conducted two-sided and considered significant when $P<.05$.

Availability of data and material All data and material presented in this article are available online:

h t t p s : / / o s f. i o / k e q r n / ? vi e w_o n 1 y = 459c7faa579748c294b876cec64c3570.

\section{Results}

\section{Experiment 1}

Participants in the L-DOPA and placebo groups did not differ significantly on STAI-T, STAI-S and ASI scores, US amplitude, US rating or reported side effects (independent sample $t$ tests, all $p>$.12: see Supplementary Table 1). A repeated measures ANOVA with stimulus as within- and group as betweensubject factor showed that groups did not differ on CRs at the end of conditioning on day 1 (stim: $F(1,68)=57.95, p<.001$, partial $\eta^{2}=.46$; group: $F(1,68)=.05, p=.83$; stim* group: $F(1,68)=.47, p=.49)$. Groups did also not differ on CRs at the beginning (stim: $F(1,68)=20.99, p<.001$, partial $\eta^{2}=.24$; group: $F(1,68)=.25, p=.62$; stim*group: $F(1,68)=.00, p=.97)$ or end (stim: $F(1,68)=12.73$, $p=.001$, partial $\eta^{2}=.16$; group: $F(1,68)=.48, p=.49$; stim* group: $F(1,68)=1.23, p=.25)$ of extinction on day 2 . Notably, post-extinction L-DOPA administration after the short extinction session in experiment 1 did not reduce CRs at test on day 3 (Fig. 2a, b; stim: $F(1,68)=16.80, p<.001$, partial $\eta^{2}=.20$; group: $F(1,68)=.12, p=.74$; stim* group: $F(1,68)=.77, p=.38)$. Similar results were obtained for FPS (Supplementary Fig. 1), online fear ratings (Supplementary Fig. 2) and pre- and post-phase US expectancy ratings on all days (Supplementary Fig. 3).

However, in line with the hypothesis that extinction success determines the effect of L-DOPA, multiple regression analysis revealed a significant interaction between CRs at the end of extinction learning and group $\left(\beta_{\text {endfear*group }}=.45\right.$, $\mathrm{SE}=.18, t(64)=2.69, p=.009$; Fig. 3a, b). We employed analysis of simple slopes (cf. Aiken and West 1991) that takes data from all participants into account to probe the nature of this interaction. As visible in Fig. 3b and confirmed by simple slope analysis, differential CRs at the end of extinction and differential CRs at test were significantly positively related in L-DOPA-treated participants $\left(\beta_{\text {endfear }}=.60, \mathrm{SE}=.15, t(64)=\right.$ $4.13, p<.001)$. That is, CRs at test were low in L-DOPAtreated participants with low CRs at the end of extinction, but high in L-DOPA-treated participants with high CRs at the end of extinction. Critically, there was no relationship between CRs at the end of extinction and test in the placebo 
a

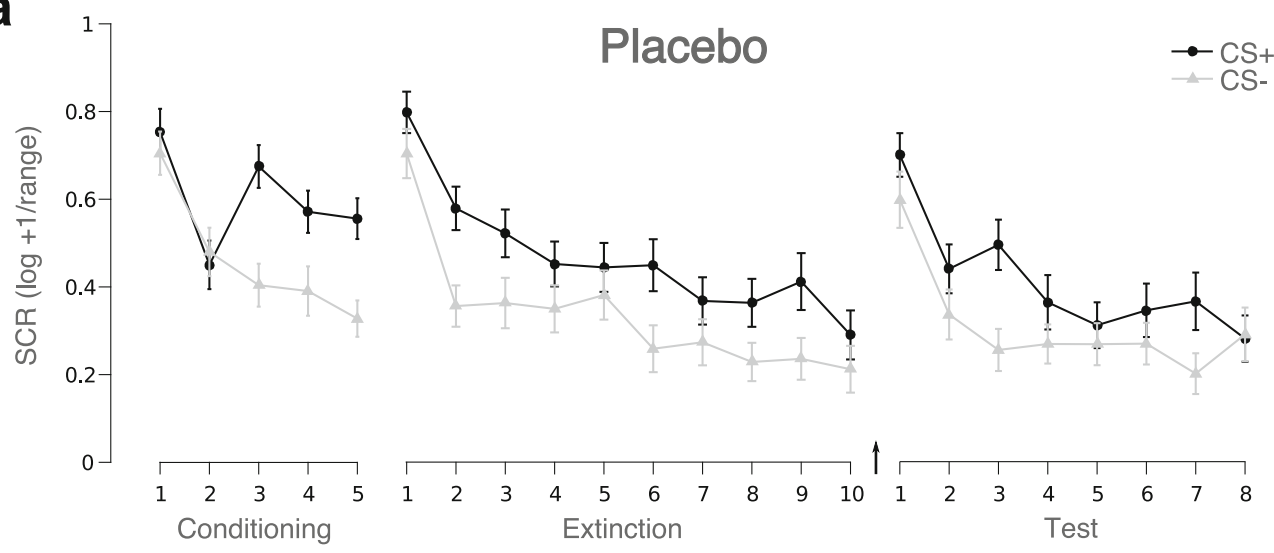

b

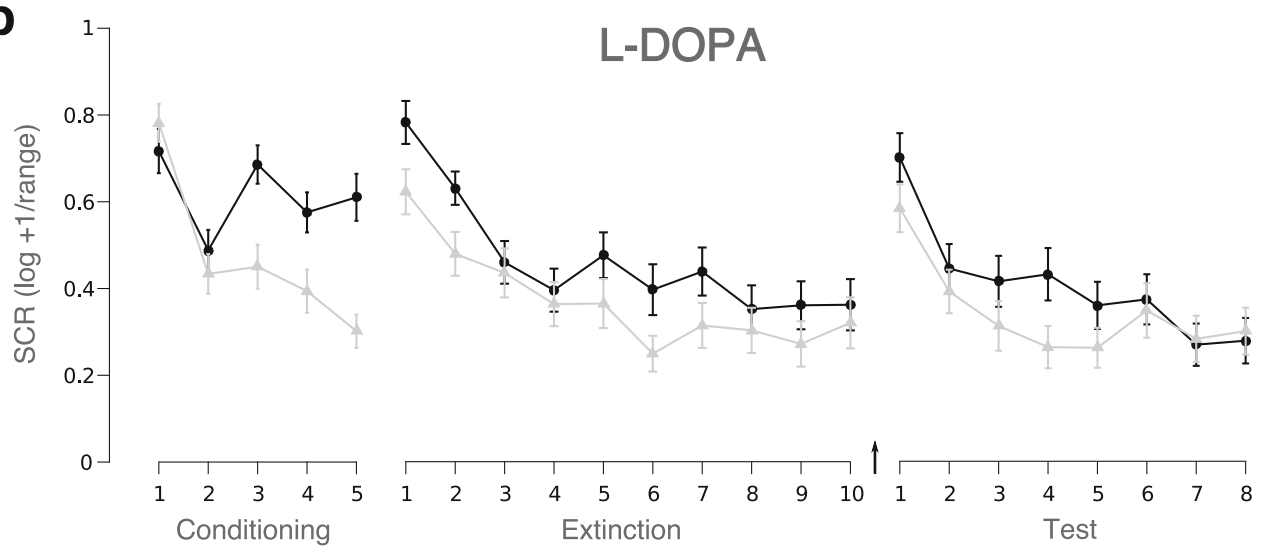

C
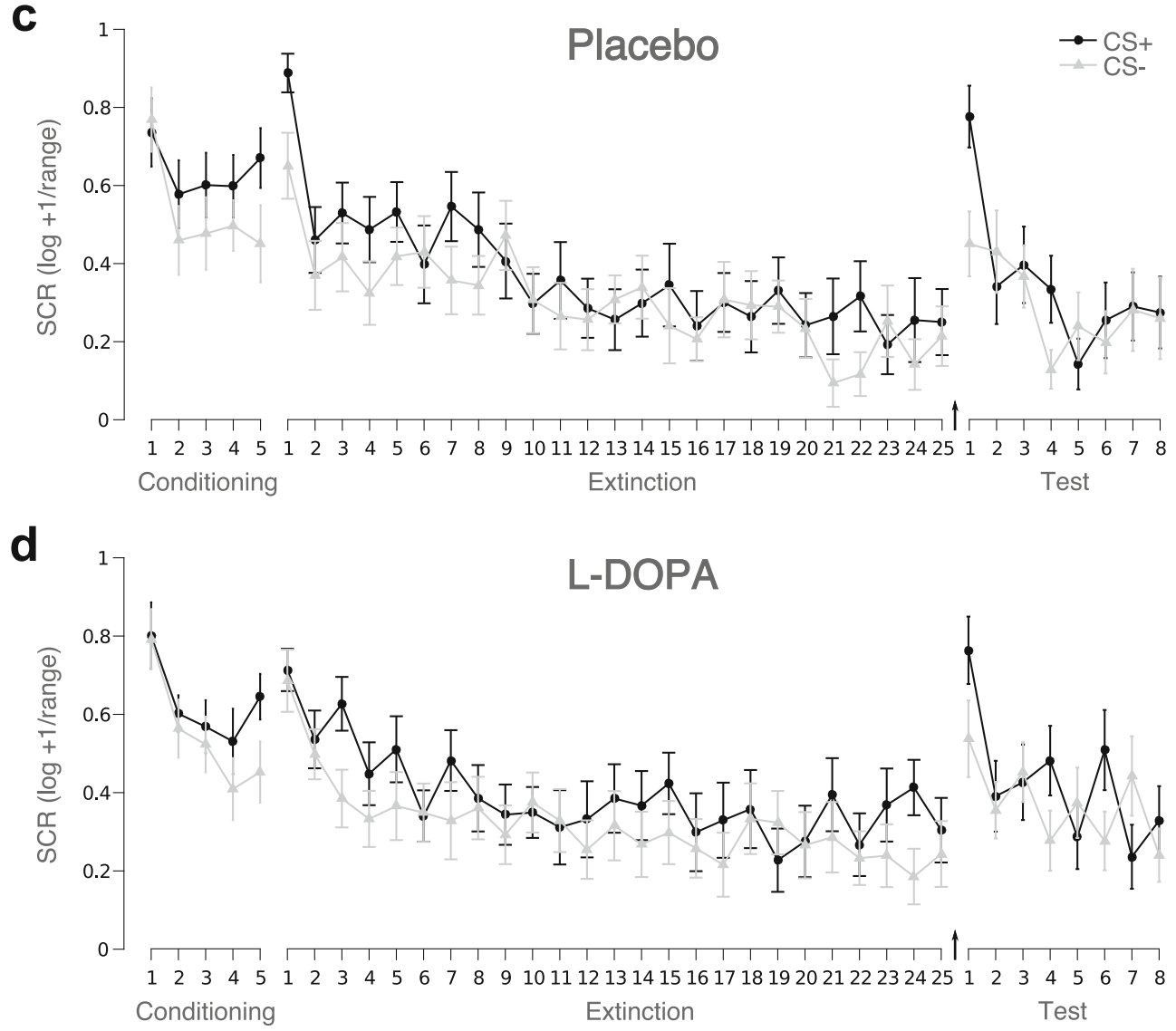
Fig. 2 Trial by trial SCRs to CS+ and CS- during conditioning on day 1, extinction on day 2 and test on day 3. Skin conductance responses of a placebo $(N=35)$ and b L-DOPA-treated $(N=35)$ participants in experiment 1 did not differ significantly in any of the experimental phases. In contrast to previous findings (Gerlicher et al. 2018), there was no of effect of L-DOPA administration after short extinction on group average CS+ compared to CS- SCRs during test on day 3. Similarly, in experiment 2 groups treated with c placebo $(N=14)$ and $\mathbf{d}$ L-DOPA $(N=16)$ after a long extinction session did also not differ significantly in any of the experimental phases. Note, however, that the intended manipulation of prolonging extinction in order to fully reduce CRs was not successful and the long extinction session did not result in a complete reduction of differential (CS+ > CS-) SCRs at the end of the extinction session. Error bars depict standard error of the mean. Arrows indicate time point of drug administration group $\left(\beta_{\text {endfear }}=.12, \mathrm{SE}=.11, t(64)=1.13, p=.26\right.$; Fig. 3a), indicating that $\mathrm{CRs}$ at the end of extinction do not predict CRs at test under placebo (normal) conditions.

Interestingly, there was no group-specific relation between CRs at the end of extinction and CRs at test as assessed by FPS or fear ratings (FPS: $\beta_{\text {endfear*group }}=-.12, \mathrm{SE}=.16$, $t(57)=-.75, p=.46$; fear ratings: $\beta_{\text {endfear*group }}=-.05$, $\mathrm{SE}=.11, t(73)=-.46, p=.65)$.

In all regression analyses, omitting CRs at the end of conditioning on day 1 and CRs at the beginning of extinction on day 2 as covariates did not change the results (data not shown). Additional exploratory analyses trying to predict CRs at test from the difference between CRs at the beginning and end of extinction did not reveal any significant relation in
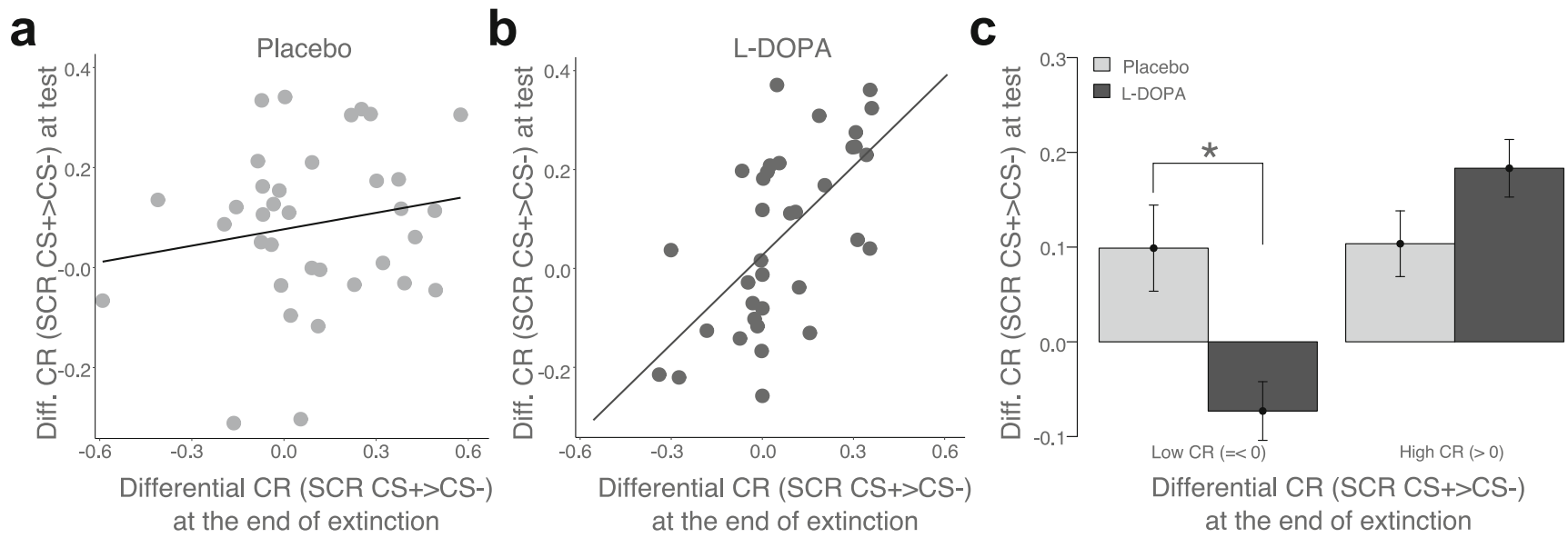

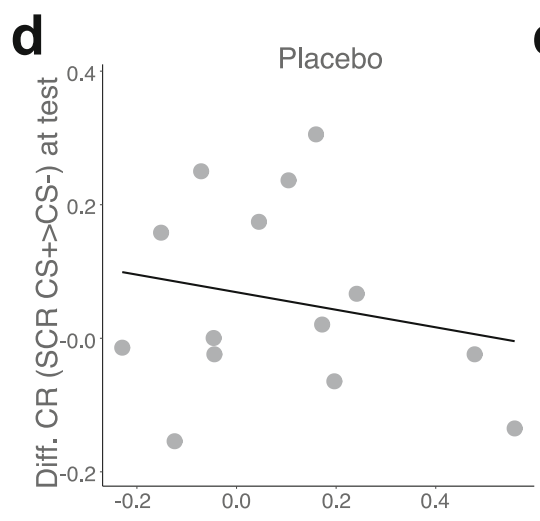

Differential CR (SCR CS+>CS-) at the end of extinction

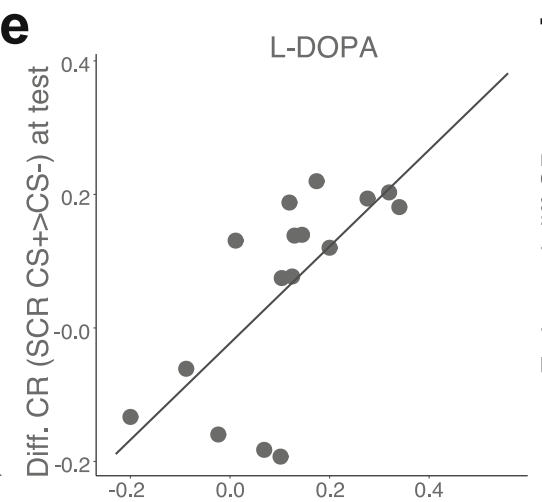

Differential CR (SCR CS+>CS-) at the end of extinction

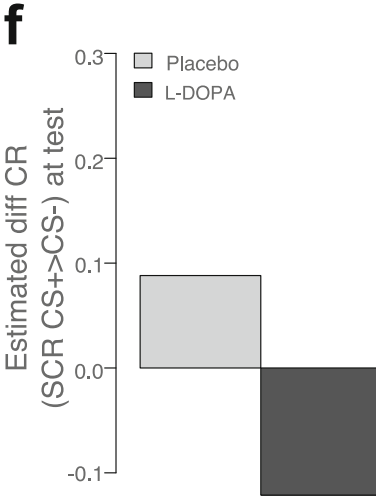

$-1 \mathrm{SD}$

$+12 D$

at the end of extinction
Fig. 3 Relationship between CRs at the end of extinction on day 2 and CRs during test on day 3 in placebo and L-DOPA-treated participants in experiments 1 (upper panels) and 2 (lower panels). a Whereas there was no significant relationship between CRs (SCR CS+ > CS-) at the end of extinction on day 2 and CRs (SCR CS+ $>$ CS-) at test on day 3 in placebotreated participants $(N=35)$, b there was a significant positive relationship after post-extinction L-DOPA administration $(N=35)$ in experiment 1. c Post hoc comparison of L-DOPA $(N=16)$ compared to placebotreated individuals $(N=12)$ with a complete reduction of CRs ((SCR $\mathrm{CS}+>\mathrm{CS}-) \leq 0$, 'low CR' in the panel) revealed that L-DOPA significantly improved extinction memory retrieval after successful withinsession extinction. In contrast L-DOPA $(N=19)$ compared to placebo- treated $(N=23)$ individuals with high $\mathrm{CRs}((\mathrm{SCR} \mathrm{CS}+>\mathrm{CS}-)>0)$ at the end of extinction showed a trend towards impaired extinction memory retrieval. d Replicating the effect of experiment 1 , also in experiment 2 not placebo $(N=14)$, but e only L-DOPA-treated participants $(N=16)$ showed a significant positive relationship between CRs (SCR CS+ > CS-) at the end of extinction on day 2 and CRs (SCR CS+ > CS-) at test at test $24 \mathrm{~h}$ later. f Point estimates of CRs (SCR CS+ > CS-) at test for participants with low (mean $-1 \mathrm{SD}$ ) and high (mean $+1 \mathrm{SD}) \mathrm{CR}$ at the end of extinction show that also in experiment 2, L-DOPA was only beneficial in individuals showing successful within-session extinction, but may have detrimental effects after non-complete within-session extinction. Error bars depict standard error of the mean 
either treatment group $\left(\beta_{\text {delta*group }}=-.21, \mathrm{SE}=.12, t(66)=-\right.$ $1.67, p=.10)$, suggesting that the relative reduction of CRs from the beginning to the end of extinction (i.e. within-session habituation) might be a comparatively less important determinant of the L-DOPA effect compared to absolute levels of fear responding at the end of extinction.

In order to exclude that inter-individual differences in differential CRs at the end of extinction were the result of differences in US magnitude, perceived US intensity, initial fear acquisition, start-fear, STAI-T, ASI or day 2 STAI-S scores, we directly correlated extinction end-fear with these measures. However, neither of the measures showed a significant relation to extinction end-fear (all $|r|$ 's $<.21$, all $p$ 's $>.08$, not corrected for multiple tests).

\section{Experiment 2}

Relative to experiment 1 , we had prolonged extinction learning from 10 to 25 trials in experiment 2 in order to achieve low CRs at the end of extinction in all subjects. In experiment 2, placebo and L-DOPA groups did also not differ significantly in respect to STAI-T, STAI-S, ASI scores, US amplitude, US rating or reported side effects (all $t<.81$, all $p>.43$; see Supplementary Table 2). Repeated measures ANOVA confirmed that groups did not differ in CRs at the end of conditioning on day 1 (stim: $F(1,28)=13.99, p=.001$, partial $\eta^{2}=.33$; group: $F(1,28)=.32, p=.57$; stim*group: $F(1,28)=.00, p=.97)$ or CRs at the beginning of extinction (stim: $F(1,28)=37.71, p<.001$, partial $\eta^{2}=.53$; group: $F(1,28)=.00, p=.97$; stim* group: $F(1,28)=.43, p=.52)$. However, unexpectedly, prolonged extinction learning did not lead to a complete reduction of CRs, but there was still a significant effect of stimulus at the end of extinction (stim: $F(1,28)=8.81, p=.006$, partial $\left.\eta^{2}=.24\right)$ in both groups (group: $F(1,28)=1.40, p=.25$; stim* group: $F(1,28)=.09$, $p=.77$ ). In addition, as in experiment 1 , there was an absence of an unqualified (average group-level) effect of L-DOPA on CRs during test on day 3 (stim: $F(1,28)=4.53, p=.04$, partial $\eta^{2}=.14$; group: $F(1,28)=1.09, p=.31$; stim*group: $F(1,28)=.00, p=.98)$. Similar results were obtained for FPS (Supplementary Fig. 4), online fear ratings (Supplementary Fig. 5) and pre- and post-phase US expectancy ratings during all experimental phases (Supplementary Fig. 6). We therefore applied the same analysis strategy as in experiment 1 and tested whether extinction success moderated the effect of LDOPA on CRs at test.

Confirming the results of experiment 1 , multiple regression analysis again revealed that extinction success affects the effect of L-DOPA, as indicated by a significant interaction between group and CRs at the end of extinction $\left(\beta_{\text {endfear*group }}=\right.$ $1.03, \mathrm{SE}=.33, t(24)=3.09, p=.005$; Fig. 3d, e). Follow-up analysis of simple slopes (cf. Aiken and West 1991) that takes data from all participants into account showed that CRs at the end of extinction and CRs during test were positively related in the L-DOPA group (Fig. 3e; $\beta_{\text {endfear }}=.98, \mathrm{SE}=.30, t(24)=$ $3.22, p=.004)$. That is, L-DOPA preserved low fear in participants with successful extinction and high fear in participants with non-successful fear reduction on day 2 . As in experiment 1 , there was no significant relationship between CRs at the end of extinction and CRs at test in the placebo group $\left(\beta_{\text {endfear }}=\right.$ $-.07, \mathrm{SE}=.16, t(24)=-.38, p=.64)$ (Fig. 3d).

There were again no comparable effects on FPS $\left(\beta_{\text {endfear*group }}=-.24, \mathrm{SE}=.36, t(25)=-.68, p=.50\right)$ and fear ratings $\left(\beta_{\text {endfear* group }}=-.25, \mathrm{SE}=.19, t(26)=-1.29\right.$, $p=.21$ ), and omitting initial fear acquisition and start-fear as covariates in all regression analyses did not change the results (data not shown). The moderating effect of the difference between CRs at the beginning and end of extinction was non-

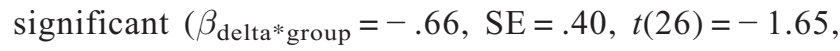
$p=.11$ ), confirming that within-session reduction of CRs is not a robust predictor of the effect of L-DOPA on CRs at test.

We again tested whether we could identify any predictor of successful extinction learning. However, US magnitude, US intensity ratings, STAI-T, ASI and STAI-S scores on day 2, differential CRs at the end of conditioning or the beginning of extinction, were not related to differential CRs at the end of extinction learning (all $|r|$ 's $<.29$, all $p$ 's $>.12$ ).

\section{The effect of L-DOPA vs. placebo in participants with successful and non-successful extinction}

To further qualify the results, we finally tested whether LDOPA is superior to placebo administration in a sub-group of participants with successful extinction only (low CR at the end of extinction: (SCR CS $+>$ CS-) $\leq 0 ; n=28$ ) in experiment 1 . Repeated measures ANOVA with stimulus as withinand group as between-subject factor revealed that after a complete reduction of CRs at the end of extinction L-DOPA compared to placebo-treated participants showed significantly reduced CRs at test on day 3 (Fig. 3c; stim: $F(1,26)=.24$, $p=.63$; stim* group: $F(1,26)=.10 .47, p=.003$, partial $\left.\eta^{2}=.29\right)$. Specifically, this effect was due to significantly smaller differential SCRs to the CS+ during test in L-DOPA compared to placebo-treated participants with successful extinction $(t(26)=2.08, p=.047$, Cohen's $d=.80$; Fig. 3f $)$. In contrast, in participants with non-successful extinction (high $\mathrm{CR}$ at the end of extinction: (SCR CS+ $>\mathrm{CS}-)>0$ ); $n=42$ ), there was no significant effect of drug administration, but there was a weak trend towards greater CRs at test after LDOPA compared to placebo administration (Fig. 3c; stim: $F(1,40)=36.97, p<.001$, partial $\eta^{2}=.48$; stim* group: $F(1,40)=2.85, p=.099)$, indicating that L-DOPA administration after non-successful extinction may even have detrimental effects on long-term expression of fear. The same results are obtained when following the approach of Aiken and West (1991) and computing regression model-based point estimates 
of CRs at test for different levels of CRs at the end of extinction for each drug group (Supplementary Fig. 7).

Given the small sample size in experiment 2 that prevents separate sub-group analyses in participants with successful extinction and non-successful extinction, we followed Aiken and West (1991) and computed model-based point estimates of CRs at test for L-DOPA and placebo-treated participants with successful (low CR at the end of extinction: mean -1 $\mathrm{SD}$ ) and non-successful (high $\mathrm{CR}$ at the end of extinction: mean $+1 \mathrm{SD}$ ) extinction. We observed the same pattern as in experiment 1: after successful extinction L-DOPA compared to placebo administration was beneficial and resulted in a significant reduction of CRs at test (Fig. $3 \mathrm{f} ; \beta_{\text {group }}=$ $-.21, \mathrm{SE}=.08, t(24)=-2.50, p=.02)$. In contrast, when administered after non-successful extinction L-DOPA was predicted to lead to significantly higher $\mathrm{CRs}$ at test than placebo administration (Fig. 3f; $\beta_{\text {group }}=.18, \mathrm{SE}=.07, t(24)=2.38$, $p=.03)$.

\section{Discussion}

In two experiments, we examined the role of inter-individual differences in extinction success on the effect of a postextinction L-DOPA administration on extinction memory retrieval $24 \mathrm{~h}$ later. In both experiments 1 and 2, a postextinction administration of L-DOPA did not result in a general reduction of conditioned fear at test. However, in line with previous findings (Smits et al. 2013a, b, 2014; Telch et al. 2014), the effect of L-DOPA on extinction memory retrieval was dependent on inter-individual differences in withinsession extinction. That is, post-extinction L-DOPA administration after successful within-session extinction (low CRs at the end of extinction as assessed by differential SCRs) was associated with low CRs at test on day 3. In contrast, postextinction L-DOPA administration after non-successful within-session extinction (high CRs at the end of extinction) was associated with high CRs at test on day 3. Critically, as previously shown (Rescorla 2006; Plendl and Wotjak 2010; Craske et al. 2014) CRs at test could not be predicted from CRs at the end of extinction in placebo-treated participants, indicating that, under normal conditions, differences in extinction memory consolidation processes occurring after extinction learning, rather than extinction learning itself, may determine whether extinction versus fear memories are retrieved.

In both experiments 1 and 2, we observed that L-DOPA administration after successful extinction results in significantly lower CRs at test than placebo administration. Thus, L-DOPA is a promising candidate as an adjunct to exposure treatments in anxiety disorders when administered after an exposure session resulting in a complete reduction of fear. In contrast, CRs at test in participants with non-successful extinction were trend-wise (experiment 1 ) or significantly greater (experiment 2) after L-DOPA compared to placebo administration. Thus, as with other pharmacological substances, it may be safer to only administer an extinction memory consolidation enhancing pharmacological treatment after an exposure session in which the practitioner can ensure that subjective fear is low.

If this thinking is correct, then translating a L-DOPA-based augmentation strategy to clinical practice ideally requires a reliable and objective marker for successful extinction, in order to determine a criterion that can inform the practitioner about the indication or contraindication for L-DOPA administration. In previous studies, subjective fear ratings were successfully used as a measure of extinction success in patients (Smits et al. 2013a, b, 2014; Telch et al. 2014). In contrast, in the present two experiments, subjective fear did not moderate the effect of L-DOPA. In a laboratory setting with healthy participants, fear ratings may, however, be more susceptible to idiosyncratic rating scale usage, demand or social desirability effects than reflecting the actual emotional state of an individual. Thus, before finally rejecting subjective fear as a marker for successful extinction, their predictive validity for clinical outcomes should be explored in more detail. In addition, skin conductance measures could also be employed in a clinical setting. Ambulatory skin conductance measurements were recently shown to reflect PTSD symptom severity (Jovanovic et al. 2018) and symptom reduction after exposure treatment (Hinrichs et al. 2017). In a case study the authors further reported that skin conductance levels (SCL) reflected the outcome of a successful exposure session (Post et al, 2017). A validation of the method in larger samples is still outstanding. Together with reports of low subjective fear, a post-exposure return of SCL to a baseline SCL level, may, thus, help to reduce the risk of an inadvertent enhancement of fear memory reconsolidation by post-exposure L-DOPA administration.

A second aim of our study was to investigate whether postextinction L-DOPA administration would also act on the expression of conditioned fear as assessed by FPS. However, in contrast to the effects of L-DOPA on SCRs in the present and other independent studies (Haaker et al. 2013; Gerlicher et al. 2018), we did not observe any effect of L-DOPA on FPS. FPS responses are thought of as a valence-specific (Hamm and Vaitl 1996) and amygdala-dependent index of conditioned fear (Davis 1992; Funayama et al. 2001; Weike et al. 2005). In contrast, SCRs are mediated by a range of different cortical and subcortical brain areas and involve the sympathetic nervous system (e.g. Dawson et al. 2007). Dissociations between SCR and FPS are commonly observed in human fear conditioning studies (Weike et al. 2005; Weike et al. 2007). Maybe due to these dissociations, many human studies investigating pharmacological manipulations of fear or extinction memory (re-)consolidation do not assess SCR and FPS simultaneously. Importantly, in those who do, differential pharmacological effects on SCR and FPS were observed, e.g. administration 
of the beta-adrenergic receptor blocker propranolol during fear memory reconsolidation eliminated fear as assessed by FPS, but left SCR unchanged (Kindt et al. 2009; Soeter and Kindt 2010, 2011, 2012; Sevenster et al. 2012). Understanding why a manipulation of fear memory reconsolidation affects FPS but not SCR, whereas the present manipulation of extinction memory consolidation showed the opposite effect, requires a better understanding of the locus of action of the pharmacological substances employed, the mapping of the different memory systems and the neural mediators of the behavioural fear expression measures in humans. Thus, a satisfying explanation for the differential effect of L-DOPA on SCR and FPS can presently not be provided. Importantly, though, previous reports show that changes in SCRs can precede changes in subjective fear report (Hodgson and Rachman, 1974). Similarly, a pharmacological disruption of fear memory reconsolidation was immediately reflected in improved approach behaviour at the first test after drug intake, whereas reports of reduced subjective fear only followed at subsequent tests (Soeter and Kindt, 2015). Thus, an important question for future research is whether the initial effect of LDOPA on differential SCRs is integrated across response systems after repeated tests of extinction memory retrieval.

A strong argument for employing L-DOPA over other pharmacological extinction enhancers is that it has been shown to be effective not only in improving extinction memory retrieval (Whittle et al. 2016) but also in reducing contextual renewal of fear in rodents (Haaker et al. 2013). Contextual renewal has been suggested to lie at the core of relapse of anxiety in patients (Vervliet et al. 2013) and is rarely prevented by pharmacological extinction enhancement (Singewald et al. 2015). In a previous study, we observed that L-DOPA may also prevent contextual renewal of fear in humans (Haaker et al. 2013). However, in this study, both conditioning and extinction were conducted during one experimental session, such that the effect of a post-extinction L-DOPA administration on extinction memory consolidation could not be differentiated from potential effects on fear memory consolidation. Thus, the question of whether L-DOPA also protects against renewal is still open and future studies should also include memory tests in contexts that provoke contextual renewal of fear (e.g. conditioning context or new context).

The question remains why the main effect of L-DOPA was stronger and less dependent on within-session extinction in our previous MRI studies (Haaker et al. 2013; Gerlicher et al. 2018) than in the present experiments. Several studies have observed an increase in cortisol and salivary alpha amylase (a marker of noradrenergic activity) from pre- to postMRI scanning (Eatough et al, 2009; Muehlhan et al, 2011; van Stegeren et al, 2006; Visser et al, 2015), indicating that placing and measuring participants in an MRI scanner can lead to a release of stress hormones, even after MRI exposure on consecutive days (Lueken et al, 2012; Peters et al, 2011).
As both cortisol (Merz et al, 2017) and noradrenaline (Abraham et al, 2012) can affect extinction learning and memory consolidation (but see Lonsdorf et al, 2014), the stronger main effect of L-DOPA can potentially be explained by an additive effect of cortisol/noradrenaline and L-DOPA in the MRI studies (Haaker et al. 2013; Gerlicher et al. 2018). This hypothesis is currently being tested in a pre-registered fMRI study (Hu et al. 2018).

To conclude, we here present and replicate evidence that a dopaminergic enhancement of extinction memory consolidation is successful in improving human extinction memory retrieval, but specific to conditions of low fear at the end of extinction. These results fall into line with reports about the dependence of DCS, yohimbine and methylene blue on the expression of fear at the end of exposure sessions and point towards a challenge common to pharmacological extinction memory enhancers. Our results emphasize the importance of limiting the use of extinction enhancers to exposure sessions with good fear reduction and stress the need for a reliable marker-i.e. a measure and a criterion to assess successful extinction-in order to prevent an inadvertent enhancement of fear.

Acknowledgements We thank A. Schick for blinding the drugs and N. Schappe and L. Brotte for the assistance with recruitment and data collection.

Funding R.K. receives funding from the German Research Foundation (Deutsche Forschungsgemeinschaft DFG CRC1193, subproject B01 and $\mathrm{C} 01$ ), the State of Rhineland-Palatinate (DRZ program) and the Boehringer Ingelheim Fonds (DRZ program); O.T. receives funding from the German Federal Ministry of Education and Research (Bundesministerium für Bildung und Forschung), the German Research Foundation (Deutsche Forschungsgemeinschaft DFG CRC1193, subproject C04) and the State of Rhineland-Palatinate (DRZ program).

\section{Compliance with ethical standards}

Conflict of interest The authors declare that they have no conflict of interest.

Open Access This article is distributed under the terms of the Creative Commons Attribution 4.0 International License (http:// creativecommons.org/licenses/by/4.0/), which permits unrestricted use, distribution, and reproduction in any medium, provided you give appropriate credit to the original author(s) and the source, provide a link to the Creative Commons license, and indicate if changes were made.

\section{References}

Abraham AD, Cunningham CL, Lattal KM (2012) Methylphenidate enhances extinction of contextual fear. Learn Mem 19:67-72. https:// doi.org/10.1101/lm.024752.111

Aiken L, West S (1991) Multiple regression: testing and interpreting interactions. SAGE Publications, Inc 
Beck AT, Emery G, Greenberg RL (2005) Anxiety disorders and phobias. A cognitive perspective. Perseus Books Group, Cambridge, MA

Bolkan SS, Lattal KM (2014) Opposing effects of D-cycloserine on fear despite a common extinction duration: interactions between brain regions and behavior. Neurobiol Learn Mem 113:25-34. https://doi. org/10.1016/j.nlm.2013.12.009

Boucsein W, Fowles D, Grimnes S et al (2012) Publication recommendations for electrodermal measurements. Psychophysiol 49:10171034. https://doi.org/10.1111/j.1469-8986.2012.01384.x

Bouton ME (2004) Context and behavioral processes in extinction. Learn Mem 11:485-494. https://doi.org/10.1101/lm.78804

Bouton ME, Vurbic D, Woods AM (2008) D-cycloserine facilitates context-specific fear extinction learning. Neurobiol Learn Mem 90:504-510. https://doi.org/10.1016/j.nlm.2008.07.003

Cover KK, Maeng LY, Lebrón-Milad K, Milad MR (2014) Mechanisms of estradiol in fear circuitry: implications for sex differences in psychopathology. Transl Psychiatry 4:e422. https://doi.org/10.1038/tp. 2014.67

Craske MG, Kircanski K, Zelikowsky M, Mystkowski J, Chowdhury N, Baker A (2008) Optimizing inhibitory learning during exposure therapy. Behav Res Ther 46:5-27. https://doi.org/10.1016/j.brat. 2007.10.003

Craske MG, Treanor M, Conway CC, Zbozinek T, Vervliet B (2014) Maximizing exposure therapy: an inhibitory learning approach. Behav Res Ther 58:10-23. https://doi.org/10.1016/j.brat.2014.04. 006

Davis M (1992) The role of the amygdala in fear-potentiated startle: implications for animal models of anxiety. Trends Pharmacol Sci 13:35-41. https://doi.org/10.1016/0165-6147(92)90014-W

Dawson ME, Schell AM, Filion DL (2007) The electrodermal system. In: Cacioppo JT, Tassinary LG, Berntson GG (eds) Handbook of psychophysiology, 3rd edn. Cambridge University Press, New York, NY, pp 159-181

de Kleine RA, Smits JAJ, Hendriks G-J, Becker ES, van Minnen A (2015) Extinction learning as a moderator of d-cycloserine efficacy for enhancing exposure therapy in posttraumatic stress disorder. J Anxiety Disord 34:63-67. https://doi.org/10.1016/j.janxdis.2015. 06.005

Eatough EM, Shirtcliff EA, Hanson JL, Pollak SD (2009) Hormonal reactivity to MRI scanning in adolescents. Psychoneuroendocrinology 34: 1242-1246. https://doi.org/10.1016/j.psyneuen.2009.03.006

Eisenberg M, Kobilo T, Berman DE, Dudai Y (2003) Stability of retrieved memory: inverse correlation with trace dominance. Science 301:1102-1104. https://doi.org/10.1126/science.1086881

Faul F, Erdfelder E, Lang A-G, Buchner A (2007) G*Power 3: a flexible statistical power analysis program for the social, behavioral, and biomedical sciences. Behav Res Methods 39:175-191

Fitzgerald PJ, Seemann JR, Maren S (2014) Can fear extinction be enhanced? A review of pharmacological and behavioral findings. Brain Res Bull 105:46-60. https://doi.org/10.1016/j.brainresbull. 2013.12.007

Fridlund AJ, Cacioppo JT (1986) Guidelines for human electromyographic research. Psychophysiology 23:567-589

Funayama ES, Grillon C, Davis M, Phelps EA (2001) A double dissociation in the affective modulation of startle in humans: effects of unilateral temporal lobectomy. J Cogn Neurosci 13:721-729. https://doi.org/10.1162/08989290152541395

Gerlicher AMV, Tüscher O, Kalisch R (2018) Dopamine-dependent prefrontal reactivations explain long-term benefit of fear extinction. Nat Commun 9:4294. https://doi.org/10.1038/s41467-018-06785-y

Haaker J, Gaburro S, Sah A, Gartmann N, Lonsdorf TB, Meier K, Singewald N, Pape HC, Morellini F, Kalisch R (2013) Single dose of L-DOPA makes extinction memories context-independent and prevents the return of fear. Proc Natl Acad Sci U S A 110:E2428E2436. https://doi.org/10.1073/pnas.1303061110
Haaker J, Lonsdorf TB, Kalisch R (2015) Effects of post-extinction LDOPA administration on the spontaneous recovery and reinstatement of fear in a human fMRI study. Eur Neuropsychopharmacol 25:1544-1555. https://doi.org/10.1016/j.euroneuro.2015.07.016

Hamm AO, Vaitl D (1996) Affective learning: awareness and aversion. Psychophysiology 33:698-710. https://doi.org/10.1111/j.14698986.1996.tb02366.x

Hinrichs R, Michopoulos V, Winters S, Rothbaum AO, Rothbaum BO, Ressler KJ, Jovanovic T (2017) Mobile assessment of heightened skin conductance in posttraumatic stress disorder. Depress Anxiety 34:502-507. https://doi.org/10.1002/da.22610

Hodgson R, Rachman S (1974) II. Desynchrony in measures of fear. Behav Res Ther 12:319-326. https://doi.org/10.1016/00057967(74)90006-0

Hu C-P, Andres E, Gerlicher AMV et al (2018) Dopamine-dependent prefrontal reactivations explain long-term benefit of fear extinction: a direct replication attempt. Nat Commun 9(1):4294

Jovanovic T, Hinrichs R, van Rooij S, et al. (2018) 229. Psychophysiological biomarkers predicting the development of PTSD: an emergency department prospective longitudinal study. In: Biological psychiatry. p S92

Kindt M, Soeter M, Vervliet B (2009) Beyond extinction: erasing human fear responses and preventing the return of fear. Nat Neurosci 12: 256-258. https://doi.org/10.1038/nn.2271

King G, Graham BM, Richardson R (2018) Effects of D-cycloserine on individual differences in relapse of fear. Prog NeuroPsychopharmacol Biol Psychiatry 84:115-121. https://doi.org/10. 1016/j.pnpbp.2018.02.005

Lebron-Milad K, Milad MR (2012) Sex differences, gonadal hormones and the fear extinction network: implications for anxiety disorders. Biol Mood Anxiety Disord 2:3. https://doi.org/10.1186/2045-53802-3

Lee JLC, Milton AL, Everitt BJ (2006) Reconsolidation and extinction of conditioned fear: inhibition and potentiation. J Neurosci 26:1005110056. https://doi.org/10.1523/JNEUROSCI.2466-06.2006

Lonsdorf TB, Haaker J, Fadai T, Kalisch R (2014) No evidence for enhanced extinction memory consolidation through noradrenergic reuptake inhibitiondelayed memory test and reinstatement in human fMRI. Psychopharmacology 231:1949-1962. https://doi.org/10. 1007/s00213-013-3338-8

Lueken U, Muehlhan M, Evens R, Wittchen HU, Kirschbaum C (2012) Within and between session changes in subjective and neuroendocrine stress parameters during magnetic resonance imaging: A controlled scanner training study. Psychoneuroendocrinology 37:12991308. https://doi.org/10.1016/j.psyneuen.2012.01.003

Lykken DT, Venables PH (1971) Direct measurement of skin conductance: a proposal for standardization. Psychophysiology 8:656672. https://doi.org/10.1111/j.1469-8986.1971.tb00501.x

McGaugh JL (2000) Memory - a century of consolidation. Science 287: 248-251. https://doi.org/10.1126/science.287.5451.248

Merlo E, Milton AL, Goozée ZY et al (2014) Reconsolidation and extinction are dissociable and mutually exclusive processes: behavioral and molecular evidence. J Neurosci 34:2422-2431. https://doi. org/10.1523/JNEUROSCI.4001-13.2014

Merz CJ, Hamacher-Dang TC, Stark R, Wolf OT, Hermann A (2018) Neural Underpinnings of Cortisol Effects on Fear Extinction. Neuropsychopharmacology 43:384-392. https://doi.org/10.1038/ npp.2017.227

Muehlhan M, Lueken U, Wittchen HU, Kirschbaum C (2011) The scanner as a stressor: evidence from subjective and neuroendocrine stress parameters in the time course of a functional magnetic resonance imaging session. Int J Psychophysiol 79:118-126. https://oi.org/ 10.1016/j.ijpsycho.2010.09.009

Nader K, Schafe GE, Le Doux JE (2000) Fear memories require protein synthesis in the amygdala for reconsolidation after retrieval. Nature 406:722-726. https://doi.org/10.1038/35021052 
Ney LJ, Wade M, Reynolds A, Zuj DV, Dymond S, Matthews A, Felmingham KL (2018) Critical evaluation of current data analysis strategies for psychophysiological measures of fear conditioning and extinction in humans. Int J Psychophysiol 134:95-107. https:// doi.org/10.1016/j.ijpsycho.2018.10.010

Otto MW, Kredlow MA, Smits JAJ, Hofmann SG, Tolin DF, de Kleine RA, van Minnen A, Evins AE, Pollack MH (2016) Enhancement of psychosocial treatment with D-cycloserine: models, moderators, and future directions. Biol Psychiatry 80:274-283. https://doi.org/10. 1016/j.biopsych.2015.09.007

Peters S, Cleare AJ, Papadopoulos A, Fu CH (2011) Cortisol responses to serial MRI scans in healthy adults and in depression. Psychoneuroendocrinology 36:737-741. https://doi.org/10.1016/j. psyneuen.2010.10.009

Plendl W, Wotjak CT (2010) Dissociation of within- and between-session extinction of conditioned fear. J Neurosci 30:4990-4998. https://doi. org/10.1523/JNEUROSCI.6038-09.2010

Post LM, Michopoulos V, Stevens JS, Reddy R, Maples JL, Morgan JR, Rothbaum AO, Jovanovich T, Ressler KJ, Rothbaum BO (2017) Psychological and psychobiological responses to immediate early intervention in the emergency department: Case report of one-session exposure therapy for the prevention of PTSD. Pract Innov (Wash D C) 2:55-65. https://doi.org/10.1037/pri0000043

Rescorla RA (2006) Deepened extinction from compound stimulus presentation. J Exp Psychol Anim Behav Process 32:135-144. https:// doi.org/10.1037/0097-7403.32.2.135

Rey CD, Lipps J, Shansky RM (2014) Dopamine D1 receptor activation rescues extinction impairments in low-estrogen female rats and induces cortical layer-specific activation changes in prefrontalamygdala circuits. Neuropsychopharmacology 39:1282-1289. https://doi.org/10.1038/npp.2013.338

Sevenster D, Beckers T, Kindt M (2012) Retrieval per se is not sufficient to trigger reconsolidation of human fear memory. Neurobiol Learn Mem 97:338-345. https://doi.org/10.1016/j.nlm.2012.01.009

Sevenster D, Beckers T, Kindt M (2014) Fear conditioning of SCR but not the startle reflex requires conscious discrimination of threat and safety. Front Behav Neurosci 8:32. https://oi.org/10.3389/fnbeh. 2014.00032

Singewald N, Schmuckermair C, Whittle N, Holmes A, Ressler KJ (2015) Pharmacology of cognitive enhancers for exposure-based therapy of fear, anxiety and trauma-related disorders. Pharmacol Ther 149:150-190. https://doi.org/10.1016/j.pharmthera.2014.12. 004

Smits JAJ, Rosenfield D, Davis ML, Julian K, Handelsman PR, Otto MW, Tuerk P, Shiekh M, Rosenfield B, Hofmann SG, Powers MB (2014) Yohimbine enhancement of exposure therapy for social anxiety disorder: a randomized controlled trial. Biol Psychiatry 75:840 846. https://doi.org/10.1016/j.biopsych.2013.10.008

Smits JAJ, Rosenfield D, Otto MW, Powers MB, Hofmann SG, Telch MJ, Pollack MH, Tart CD (2013b) D-Cycloserine enhancement of fear extinction is specific to successful exposure sessions: evidence from the treatment of height phobia. Biol Psychiatry 73:1054-1058. https://doi.org/10.1016/j.biopsych.2012.12.009

Smits JAJ, Rosenfield D, Otto MW, Marques L, Davis ML, Meuret AE, Simon NM, Pollack MH, Hofmann SG (2013a) D-Cycloserine enhancement of exposure therapy for social anxiety disorder depends on the success of exposure sessions. J Psychiatr Res 47:1455-1461. https://doi.org/10.1016/j.jpsychires.2013.06.020

Soeter M, Kindt M (2010) Dissociating response systems: erasing fear from memory. Neurobiol Learn Mem 94:30-41. https://doi.org/10. 1016/j.nlm.2010.03.004
Soeter M, Kindt M (2011) Disrupting reconsolidation: pharmacological and behavioral manipulations. Learn Mem 18:357-366. https://doi. org/10.1101/lm.2148511

Soeter M, Kindt M (2012) Stimulation of the noradrenergic system during memory formation impairs extinction learning but not the disruption of reconsolidation. Neuropsychopharmacology 37:1204-1215. https://doi.org/10.1038/npp.2011.307

Soeter M, Kindt M (2015) An abrupt transformation of phobic behavior after a post-retrieval amnesic agent. Biol Psychiatry 78:880-886. https://doi.org/10.1016/j.biopsych.2015.04.006

Spielberger CD, Gorsuch RL, Lushene RE (1970) Manual for the StateTrait Anxiety Inventory. Consulting Psychologists Press

Taylor S, Zvolensky MJ, Cox BJ, Deacon B, Heimberg RG, Ledley DR, Abramowitz JS, Holaway RM, Sandin B, Stewart SH, Coles M, Eng W, Daly ES, Arrindell WA, Bouvard M, Cardenas SJ (2007) Robust dimensions of anxiety sensitivity: development and initial validation of the Anxiety Sensitivity Index-3. Psychol Assess 19:176-188. https://doi.org/10.1037/1040-3590.19.2.176

Telch MJ, Bruchey AK, Rosenfield D, Cobb AR, Smits J, Pahl S, Gonzalez-Lima F (2014) Effects of post-session administration of methylene blue on fear extinction and contextual memory in adults with claustrophobia. Am J Psychiatry 171:1091-1098. https://doi. org/10.1176/appi.ajp.2014.13101407

van Stegeren A, Rohleder N, Everaerd W, Wolf OT (2006) Salivary alpha amylase as marker for adrenergic activity during stress: Effect of betablockade. Psychoneuroendocrinology 31:137-141. https://doi. org/10.1016/j.psyneuen.2005.05.012

Vervliet B, Craske MG, Hermans D (2013) Fear extinction and relapse: state of the art. Annu Rev Clin Psychol 9:215-248. https://doi.org/ 10.1146/annurev-clinpsy-050212-185542

Visser RM, Kunze AE, Westhoff B, Scholte HS, Kindt M (2015) Representational similarity analysis offers a preview of the noradrenergic modulation of long-term fear memory at the time of encoding. Psychoneuroendocrinology 55:8-20. https://doi.org/10. 1016/j.psyneuen.2015.01.021

Weber M, Hart J, Richardson R (2007) Effects of D-cycloserine on extinction of learned fear to an olfactory cue. Neurobiol Learn Mem 87:476-482. https://doi.org/10.1016/j.nlm.2006.12.010

Weike AI, Hamm AO, Schupp HT, Runge U, Schroeder HW, Kessler C (2005) Fear conditioning following unilateral temporal lobectomy: dissociation of conditioned startle potentiation and autonomic learning. J Neurosci 25:11117-11124. https://doi.org/10.1523/ JNEUROSCI.2032-05.2005

Weike AI, Schupp HT, Hamm AO (2007) Fear acquisition requires awareness in trace but not delay conditioning. Psychophysiology 44:170-180. https://doi.org/10.1111/j.1469-8986.2006.00469.x

Whittle N, Maurer V, Murphy C, Rainer J, Bindreither D, Hauschild M, Scharinger A, Oberhauser M, Keil T, Brehm C, Valovka T, Striessnig J, Singewald N (2016) Enhancing dopaminergic signaling and histone acetylation promotes long-term rescue of deficient fear extinction. Transl Psychiatry 6:e974. https://doi.org/10.1038/tp. 2016.231

Publisher's note Springer Nature remains neutral with regard to jurisdictional claims in published maps and institutional affiliations. 